\title{
Identificação de locais com potencial de transmissão de dengue em Porto Alegre através de técnicas de geoprocessamento
}

\author{
Identification of places with potential transmission of dengue fever \\ in Porto Alegre using Geographical Information Systems
}

\author{
Christovam Barcellos ${ }^{1}$, Adelaide Kreutz Pustai², Maria Angélica Weber ${ }^{2}$ \\ e Maria Regina Varnieri Brito ${ }^{2}$
}

\begin{abstract}
RESUMO
Ainda pouco se conhece sobre as condições socioambientais que favorecem a permanência do Aedes aegypti em áreas urbanas e sua capacidade de transmissão de dengue. A proposta desse trabalho é localizar os casos da doença e a presença do vetor, e identificar fatores sócio-ambientais que caracterizam esses locais, através de técnicas de geoprocessamento, procurando desenvolver um modelo de prevenção de dengue. 0 vetor foi encontrado principalmente nas zonas sul e leste da cidade, apresentando uma grande dispersão no município, enquanto a maior parte dos casos está localizada na parte central da cidade. Os setores que apresentaram casos possuem características de alta renda. Por outro lado, nos setores com a presença de vetor são verificadas a predominância de casas e boa infraestrutura de saneamento. A diferença dos padrões de distribuição de casos e vetor assegurou para 0 ano de 2002 a ausência de transmissão do vírus no município.
\end{abstract}

Palavras-chaves: Dengue. Geoprocessamento. Prevenção de doenças. Vigilância em Saúde. Análise espacial.

\begin{abstract}
The social-environmental conditions that promote the continuity of Aedes aegypti in urban areas and its capacity for dengue transmission have yet to be determined. The aim of this work was to locate dengue cases and vector presence, then to identify social-environmental factors that characterize these sites, through Geographical Information Systems (GIS), with a view to developing a dengue fever prevention model. The vector was mainly located in south and east areas of the city, with a broad dispersion, while most of the cases were located in the central region of the city. The sectors with cases present characteristics of high income. Otherwise, sectors with vector present a predominance of houses and good sanitation infrastructure. The difference between cases and vector spatial patterns ensured for 2002 an absence of dengue transmission in the city.
\end{abstract}

Key-words: Dengue fever. Geographical Information Systems. Disease prevention. Health surveillance. Spatial analysis.

A dengue teve ao longo da última década uma difusão incontrolada no território brasileiro. As estratégias de controle até 0 momento têm se mostrado pouco efetivas devido à intensiva urbanização da doença e a limitações do próprio processo de vigilância da doença ${ }^{16}$. As atividades humanas têm um papel preponderante na distribuição do vetor e na difusão do vírus. 0 fluxo populacional pode representar um fator de difusão do vírus, e em cidades de grande porte a ocupação desigual do espaço forma paisagens que podem promover estratos diferenciados de transmissão de dengue, marcadamente no que se refere à permanência de habitats favoráveis ao vetor ${ }^{4}$. Destacando as diferenças da paisagem Souza-Santos ${ }^{15}$ alerta que, os estudos devem ser conduzidos de forma a identificar métodos de vigilância para a aplicação em nível local, pois uma mesma espécie em diferentes áreas geográficas pode diferir em suas características biológicas e comportamentais. No entanto, poucos trabalhos têm se dedicado ao estudo da distribuição do dengue e do seu vetor nas áreas urbanas.

\footnotetext{
1. Departamento de Informações em Saúde da Fundação Oswaldo Cruz, Rio de Janeiro, RJ. 2. Centro de Vigilância em Saúde da Secretaria Municipal de Saúde de Porto Alegre, Porto Alegre, RS.

Endereço para correspondência: Dr. Christovam Barcellos. DIS/CICT/FIOCRUZ. Av. Brasil 4365, Manguinhos, 21045-900 Rio de Janeiro, RJ.

Telefax: 21 2290-1696,

e-mail: xris@ cict.fiocruz.br

Recebido em 4/9/2004

Aceito em $5 / 3 / 2005$
} 
A vigilância da dengue no Brasil é realizada através de dois principais sistemas de informação: 0 Sistema de Informações sobre a Febre Amarela e Dengue (SISFAD), que registra as atividades de vigilância entomológica e 0 Sistema Nacional de Agravos de Notificação ( SINAN) , que registra os casos confirmados e suspeitos da doença. Esses sistemas possuem lógicas e objetivos diferentes; dificultando o relacionamento entre seus dados. Essa vinculação é indispensável para se verificar a presença simultânea de infestação pelo vetor e casos da doença, condição para 0 surgimento de surtos de dengue. Da mesma maneira, permitiria avaliar a efetividade dos programas de controle do vetor, isto é, verificar se a eliminação de focos tem como consequiência a diminuição do número de casos. Devido ao desenho desses sistemas, os indicadores sobre vetor e doença só podem ser relacionados usando-se o município e, em alguns casos, 0 bairro, como unidade de agregação. Essas unidades espaciais possuem uma grande heterogeneidade interna e apresentam dimensões grandes demais para contemplar 0 objetivo de identificar e atuar sobre focos com poucos quilômetros de extensão. Além disso, é necessária que haja uma automatização no processo de entrada e cruzamento entre os dados entomológicos e epidemiológicos para que haja um alerta quase instantâneo quando da identificação da interseção entre a presença do vetor e casos suspeitos da doença. A proposta desse trabalho é baseada no uso de técnicas de geoprocessamento de dados de saúde com o objetivo de localizar com mais precisão os casos da doença e a presença do vetor, procurando desenvolver um modelo de prevenção da transmissão de dengue em áreas urbanas.

A região sul do Brasil possui os menores índices de casos de dengue no Brasil, sendo o Rio Grande do Sul e Santa Catarina considerados estados sem a transmissão autóctone da doença. No entanto, vários municípios desses estados vêm registrando a presença do vetor ${ }^{5}$. Durante do primeiro semestre de 2002, foram notificados 127 casos de dengue no município de Porto Alegre, todos importados e decorrentes da grave epidemia ocorrida no Rio de Janeiro e outras cidades do país no verão desse ano. Essa epidemia teve reflexos importantes na cidade, uma vez que muitas pessoas que haviam estado em cidades com epidemias retornaram infectadas. 0 problema se potencializa pela presença do mosquito Aedes aegypti na cidade.

0 objetivo desse trabalho foi a identificação de áreas de risco de transmissão autóctone de dengue, através do mapeamento dos casos importados de dengue e das medidas de infestação pelo vetor. São discutidos os determinantes da distribuição de casos e do vetor no espaço urbano e avaliados cenários potenciais de transmissão autóctone do vírus, através do exame de indicadores sócio-ambientais das áreas com presença de casos e do vetor. Esses cenários representam pistas para 0 aprimoramento das ações de vigilância de dengue em outras cidades.

\section{MATERIAL E MÉTODOS}

Neste estudo foram utilizadas duas bases de dados: os casos notificados de dengue e os dados de infestação pelo vetor. Foram considerados todos os 127 casos confirmados de dengue de pessoas com endereço fixo ou temporário em Porto Alegre, no período de janeiro a julho de 2002, contidos no SINAN. Todos esses casos foram georreferenciados através do endereço de residência, usando-se o Sistema de Informações Geográficas do município (SIGPoa) que contém o mapa digitalizado de logradouros, setores censitários ( SC) e bairros da cidade. Foram também georreferenciados os imóveis com a presença de larvas observadas durante 0 Levantamento de Índice (L) previsto como atividade de controle do vetor. Para esse processo, foi utilizado um banco de dados gerado no SISFAD pela Equipe de Controle de Zoonoses da Secretaria Municipal de Saúde. Nesse banco de dados foram digitados os endereços dos 698 focos de Aedes aegypti encontrados em Porto Alegre, no período de janeiro a julho de 2002. Foi possivel através do SIGPoa georreferenciar 664 imóveis, 0 que representa uma eficiência de $95 \%$ de localização.

Os dados pontuais de localização de casos de dengue e presença do vetor foram tratados estatisticamente através do programa Crimestat ${ }^{9}$ para 0 cálculo da média espacial e 0 traçado de elipses de desvio padrão da distribuição espacial dos eventos. Esse procedimento permite a delimitação de uma área, chamada envoltória, que contém a maior parte dos eventos.

Para cada setor censitário (SC) foram calculados 25 indicadores sócio-ambientais mais relevantes para a transmissão de dengue, incluindo condições de habitação, saneamento, renda escolaridade. Através de operações entre as camadas em ambiente de sistema de informações geográficas, foram identificados 84 SC com a presença de casos e $313 \mathrm{com}$ a presença do vetor. 0 banco de dados foi exportado para um programa de análise estatística através do qual foi calculada a média e desvio padrão dos indicadores sócio-ambientais, e realizada análise de variância para determinar a significância estatística da diferença entre médias. Foi também realizada a regressão logística tendo como desfecho a variável dicotômica presença/ausência de casos de dengue e do vetor no SC e como variáveis explicativas os indicadores sócio-ambientais.

\section{RESULTADOS E DISCUSSÃ0}

A partir do georreferenciamento de casos e domićlios com 0 vetor foi possível verificar o padrão de distribuição espacial desses eventos no município de Porto Alegre. Afigura 1 mostra a localização de domicílios com a presença do vetor e casos de dengue ocorridos no primeiro semestre de 2002 . 0 vetor foi encontrado principalmente nas zonas sul e leste da cidade, apresentando uma grande dispersão no município. A maior concentração do vetor está localizada nas encostas do Morro de Santa Teresa e da Polícia.

A maior parte dos casos de dengue está localizada na parte central da cidade, com pouca dispersão ao longo do eixo de desenvolvimento tradicional da cidade ${ }^{11}$. A figura mostra as elipses que representam as envoltórias da localização do vetor e de casos de dengue. Observa-se que a envoltória do vetor tem maior área e se localiza mais ao sul, enquanto a envoltória dos casos se localiza no centro da cidade e tem menor área. A intersecção entre as elipses mostra uma pequena área, ao longo do baixo curso do Arroio Dilúvio, onde a presença simultânea do vetor e 


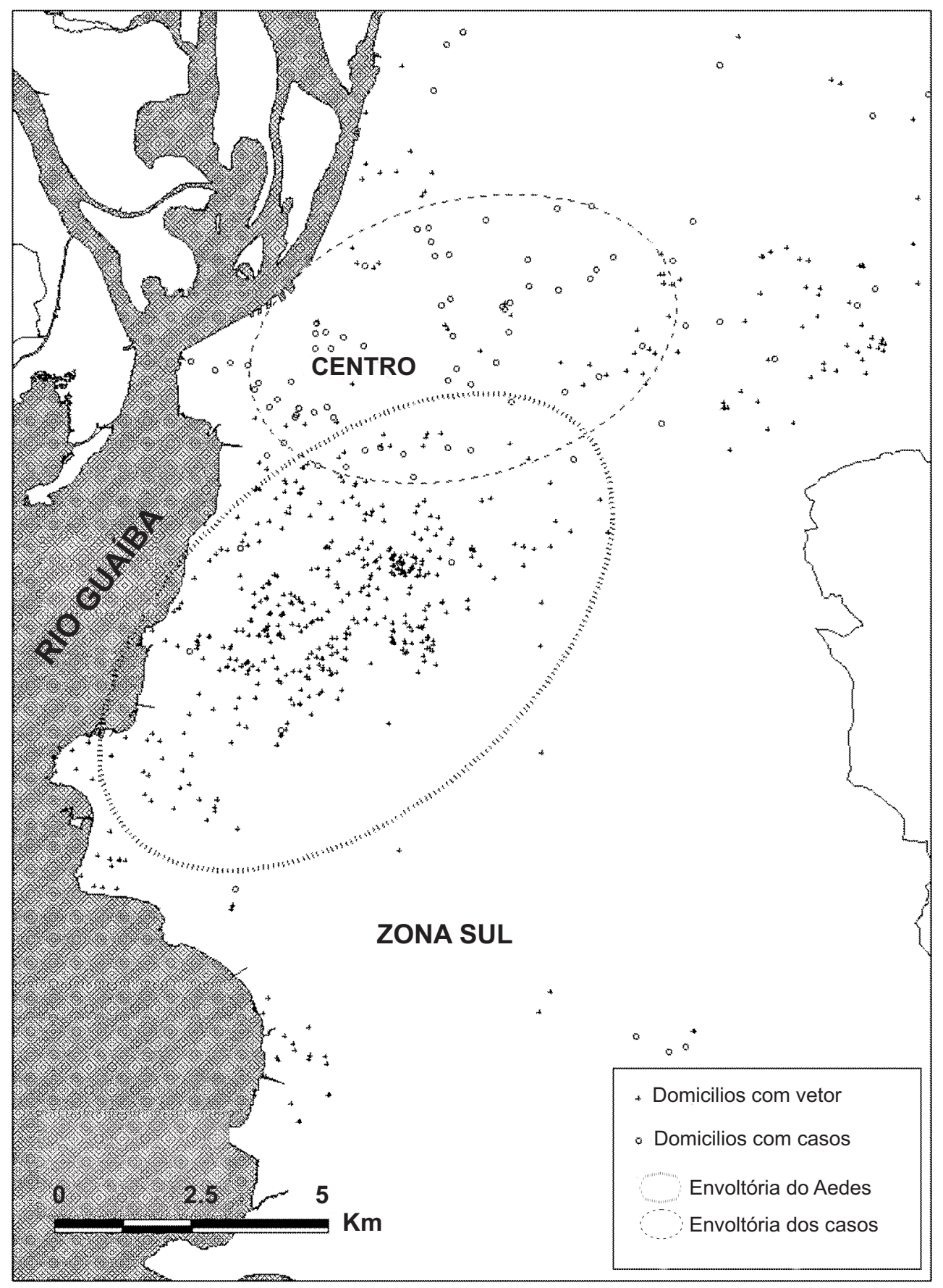

Figura 1 - Localização de domicílios com casos de dengue e com a presença de Aedes aegypti no município de Porto Alegre. As envoltórias de casos e do vetor mostram a média e o desvio padrão da localização dos pontos.

de casos de dengue poderia ter promovido a transmissão autóctone do vírus.

A diferença entre os padrões de distribuição de casos e vetor, conforme verificado acima assegurou para 0 ano de 2002 a ausência de transmissão do vírus no município. A distribuição desigual dos casos importados de doença e do vetor se deve a particularidades da cidade que influem na ecologia do vetor e no risco de adoecer por dengue. As características sócio-espaciais dos locais onde foram verificados vetores e casos foram examinadas através da análise estatística de indicadores sócio-ambientais. A Tabela 1 mostra as principais diferenças entre indicadores de SC com casos de dengue em relação ao total do município.
Tabela 1 - Média e desvio padrão de indicadores sócio-ambientais dos setores censitários com casos de dengue e com a presença do vetor em comparação ao total do município de Porto Alegre.

\begin{tabular}{|c|c|c|c|}
\hline Indicador & $\begin{array}{l}\text { Setores com } \\
\operatorname{casos}(\mathrm{n}=84)\end{array}$ & $\begin{array}{c}\text { Setores com } \\
\text { vetor }(n=313)\end{array}$ & $\begin{array}{c}\text { Total do } \\
\text { município }(n=2121)\end{array}$ \\
\hline Razão de masculinidade & $0,82 \pm 0,08^{* *}$ & $0,87 \pm 0,09$ & $0,87 \pm 0,11$ \\
\hline Baixa renda & $10,5 \pm 9,1 * *$ & $24,0 \pm 17,1$ & $22,7 \pm 19,1$ \\
\hline Instrução secundária & $46,3 \pm 21,3^{* *}$ & $26,0 \pm 20,4$ & $28,2 \pm 23,7$ \\
\hline Casas & $33,2 \pm 34,3^{* *}$ & $72,5 \pm 31,5^{* *}$ & $55,4 \pm 41,6$ \\
\hline Pessoas por domicílio & $2,79 \pm 0,52 * *$ & $3,25 \pm 0,43^{* *}$ & $3,09 \pm 0,62$ \\
\hline Rede de água & $97,8 \pm 10,3$ & $99,1 \pm 3,3^{*}$ & $97,92 \pm 10,2$ \\
\hline Rede de esgoto & $58,3 \pm 39,9 * *$ & $57,4 \pm 35,5^{* *}$ & $48,3 \pm 40,3$ \\
\hline
\end{tabular}

* Diferença significativa da média do município segundo análise de variância $p<0,05$ ** Diferença significativa da média do município segundo análise de variância, $p<0,01$. 
Os setores que apresentaram casos de dengue possuem uma população de homens significativamente menor que os demais setores da cidade. Esses setores têm menor proporção de responsáveis da família com baixa renda e maior proporção de pessoas com nível de instrução de segundo grau. Quanto aos indicadores de habitação, os setores apresentam menor proporção de casas e menos pessoas por domicílio, além de ter maior proporção de domicílios ligados à rede geral de coleta de esgotos. Esse quadro mostra o predomínio de setores de classe média entre os que apresentaram casos de dengue. A regressão logística realizada com todas as variáveis apontou a renda como a única variável para a qual se verificou uma contribuição significativa para 0 modelo de explicação da distribuição espacial da doença.

A Tabela 1 mostra também a média e desvio padrão dos indicadores sócio-ambientais de SC com a presença do vetor em relação ao total do município. As principais diferenças verificadas entre esses setores são a maior presença de casas, a maior proporção de domicílios com coleta de esgotos e abastecimento de água, bem como um maior número de pessoas por domicílio. Todas essas variáveis estão relacionadas às condições gerais de saneamento e habitação, mas ao contrário de outras cidades, a presença do vetor não está associada ao baixo índice de saneamento ${ }^{16}$. Ao contrário, 0 vetor está mais presente em áreas com alta cobertura das redes de esgoto e água. Por outro lado, a grande participação de casas ( cerca de 72\% do total de domicílios dessas áreas) e a aglomeração de pessoas no mesmo domicílio mostra uma forma de habitação particular dessas áreas da cidade, com ocupação mais recente, algumas delas caracterizadas também pela presença de favelas ${ }^{11}$. Dentre os indicadores que mostram uma contribuição significativa para o modelo logístico destacamse a proporção de casas e a cobertura da rede de coleta de esgotos. Este modelo, entretanto, apresentou alta especificidade e baixa sensibilidade e, isto é, tem capacidade somente para detectar SC sem a presença do vetor.

Vários trabalhos reconhecem o papel das condições de habitação e de renda como determinantes da intensidade de transmissão da doença mas apontam por vezes para conclusões contraditórias. Chiaravalloti Neto e cols ${ }^{3}$, em estudo sobre a colonização pelo Aedes aegypti dos 30 municípios da região de São José do Rio de Preto, concluíram que os municípios com maior densidade demográfica e menor renda apresentaram maiores riscos de transmissão de dengue. Medronho ${ }^{10}$, utilizando pioneiramente técnicas de geoprocessamento, demonstrou no Rio de Janeiro associações favoráveis de risco de dengue com áreas de favelas e principais vias de transporte. Na ilha de São Luiz foi constatada a maior soroprevalência de dengue em moradores de áreas de maior renda ${ }^{18}$. Ko e cols ${ }^{6}$ verificaram que as casas fechadas com telas representavam um fator de proteção contra a dengue para seus moradores.

0 acesso e qualidade dos serviços de abastecimento de água têm sido também apontados como determinantes da transmissão. Por um lado, a ausência desses serviços obriga moradores a estocar água em quantidade suficiente para garantir 0 suprimento, principalmente em períodos de seca. Esses reservatórios têm sido apontados como um fator favorável à procriação do vetor em áreas urbanas ${ }^{714}$. Por outro lado, essa estratégia é mais utilizada em domicílios ligados à rede geral de abastecimento. Assim, a condição mais crítica para a proliferação do vetor parece ser, mais que a ausência do serviço, a irregularidade no fornecimento de água. Os resultados obtidos nesse trabalho permitem identificar as áreas com alta cobertura dos serviços de abastecimento de água como as que apresentaram maior concentração do vetor na cidade. Em Porto Alegre, como em grande parte das áreas urbanas do Brasil, a maior parte dos domicílios estão ligados à rede geral de abastecimento de água. Nas áreas de maior concentração do vetor esse indicador alcança quase a totalidade dos domicílios (99,2\%). Como essas áreas são mais elevadas em relação ao restante da cidade, pode-se levantar a hipótese de que haja dificuldade de abastecimento em alguns domicílios devido à variação de pressão de água na canalização ${ }^{1}$. A predominância de casas nessas áreas também propicia 0 armazenamento de água em caixas de água, cisternas e tonéis. Outros recipientes relevantes para a proliferação do vetor são vasos, pneus e garrafas, que são mais freqüentes em casas que em apartamentos. Esses recipientes têm sido apontados como os de maior freqüência de presença de larvas nas atividades de inspeção predial $^{8}$. Segundo o serviço de saneamento local, as atividades domésticas com maior consumo de água são a lavagem de carros e de calçadas, bem como a rega de plantas ${ }^{11}$, que são mais comuns para esse tipo de habitação.

A comparação entre os resultados obtidos pelos modelos de distribuição de casos e do vetor mostra uma sensível diferença dos condicionantes da doença no município. Por um lado, a presença de casos está associada a áreas de maior renda. Como todos os casos notificados foram importados, a possibilidade de viajar e contrair a doença em outro estado, principalmente no Rio de Janeiro, está ligada ao modo de vida e capacidade de gasto dos moradores dessas áreas. Por outro lado, a infestação está associada às condições gerais de saneamento e principalmente ao acesso às redes de água e esgoto, em conjunção com a moradia em casas.

Essa comparação também permite identificar cenários futuros que promovam a transmissão de dengue na cidade. Como 0 vetor pode se expandir para outras áreas onde encontre condições favoráveis de proliferação, pode-se esperar uma difusão para outras áreas ocupadas também por casas que tenham acesso às redes de saneamento. Como estas condições independem da renda, as áreas mais ao norte poderão ser infestadas pelo vetor. Um outro cenário, de ainda maior risco seria dado pela permanência de pessoas infectadas na área sul da cidade, onde há maior infestação do vetor.

Apesar da presença do vetor e do surgimento de vários casos de dengue em Porto Alegre durante 0 primeiro semestre de 2002, nenhum caso foi caracterizado como autóctone. Houve mesmo uma sensível diminuição do número de casos ao longo do ano 0 que reforça a idéia de que não houve transmissão do vírus. 0 baixo risco de transmissão de dengue na cidade se deve ao contexto sócio-espacial em que ocorrem casos importados, bem como sua relativa distância às áreas mais infestadas da cidade. Enquanto os locais de maior infestação são determinados por condições gerais de saneamento e habitação, a renda limita a população potencialmente exposta ao vírus que viaja para outros estados. Essa distância física também reflete uma distância social que ironicamente protege a população geral, fornecendo uma barreira para a transmissão local da doença. 
A metodologia utilizada no presente estudo apresenta grande potencial para a vigilância da doença em cidades com baixa infestação. Através de técnicas de SIG é possível identificar mais precisamente áreas de risco, desde que haja uma atualização permanente das bases de dados de casos notificados como suspeitos de dengue, bem como da presença do vetor. Para que a metodologia proposta tenha máxima eficiência na detecção de áreas de risco de transmissão, é necessário o preenchimento adequado dos endereços nas duas bases de dados ( SISFAD e SINAN) . Em municípios que não possuem bases de logradouros para a localização de endereços, 0 GPS poderia ser adotado para a localização geográfica de focos e casos. Dessa forma, será possível adotar medidas de prevenção imediatas (eliminação dos focos) na área de influência dos casos.

0 estudo apresenta limitações, uma vez que houve intervalos de tempo grandes entre a data de início dos sintomas dos pacientes e a detecção da presença do vetor na área de residência do paciente. As classificações de risco criadas no trabalho não mostraram poder de previsão de áreas de risco, isto é, os modelos de regressão logística apresentaram baixa sensibilidade na detecção de áreas de risco.

É sabido que em locais onde se inicia a transmissão de dengue, há sempre epidemias de grande magnitude e sob forma hiperendêmica nos lugares onde circularam anteriormente um ou mais sorotipos. Existem lacunas de conhecimento disponíveis para prever, sob bases científicas, futuras ocorrências de epidemias na forma grave. Pela situação epidemiológica e entomológica de vários continentes, há evidências de agravamento do atual cenário, uma vez que há grande dificuldade em eliminar ou controlar os fatores de risco da doençą ${ }^{17}$.

Os Sistemas de Informações Geográficas têm sido usados para 0 controle de dengue visando apontar áreas de maior intensidade de transmissã $00^{213}$, identificar áreas mais vulneráveis ao vetor ${ }^{12}$ e planejar ações de controle. № entanto, a maior potencialidade desses sistemas é permitir a análise conjunta desses dados, buscando otimizar as atividades de vigilância epidemiológica e entomológica, além de programas de informação dirigidos a grupos populacionais específicos.

\section{REFERÊNCIAS BIBLIOGRÁFICAS}

1. Barcellos C, Barbosa KC, Pina MF, Magalhães MMAF, Paola JCMD, Santos SM. Interrelacionamento de dados ambientais e de saúde: Análise de risco à saúde aplicada ao abastecimento de água no Rio de Janeiro utilizando Sistemas de Informações Geográficas, Cadernos de Saúde Pública 14: 597-605, 1998
2. Barrera R, Delgado N, Jimenez M, Villalobos I, Romero I. Estratificación de riesgo en una ciudad hiperendémica para dengue hemorragico. Revista Panamericana de Salud Publica 8:225-233, 2000.

3. Chiaravalloti Neto FC, Moraes MS, Fernades MA. Avaliação dos resultados de atividades de incentivo à participação da comunidade no controle da denque em um bairro periférico do Município de São José do Rio Preto, São Paulo, e da relação entre conhecimentos e práticas desta população. Cadernos de Saúde Pública 14( supl 2):101-109, 1998.

4. Costa AIP, Natal D. Geographical distribution of dengue and socioeconomic factors in an urban locality in Southeastern Brazil. Revista de Saúde Pública 32: 232-236, 1998.

5. Fundação Nacional de Saúde. Programa Nacional de Controle da Dengue. Fundação Nacional de Saúde, Brasília, 2002.

6. Ko YC, Chen MJ, Yeh SM. The predisposing and protective factors against dengue virus transmission by mosquito vector. American Journal of Epidemiology 136:214-220, 1992.

7. Koopman JS, Prevots DR, Vaca Marin MA, Gomez Dantes H, Zarate Aquino ML, Longini IM Jr, Sepulveda Amor J. Determinants and predictors of dengue infection in Mexico. American Journal of Epidemiology 133:1168-1178, 1991.

8. Lammerhirt CB, Kieling E, Bavaresco FP, Dornelles MB. Estudo da distribuição do Aedes aegypti e Aedes albopictus no Rio Grande do Sul, 1995 a 2001. Monografia. Curso de especialização em epidemiologia. Escola de Saúde Pública do Rio Grande do Sul. Porto Alegre, 2002.

9. Levine, N. CrimeStat II: A Spatial Statistics Program for the Analysis of Crime Incident Locations. Ned Levine \& Associates, Houston, TX, and the National Institute of Justice, Washington, DC. 2002.

10. Medronho RA. Geoprossessamento e saúde uma nova abordagem do espaço no processo saúde doença. Editora . FIOCRUZ/Cict/Nect. Rio de Janeiro, 1995.

11. Menegat R. Atlas Ambiental de Porto Alegre. Ed. Universidade, Porto Alegre, 1998.

12. Mercado-Hernandez R, Fernandez-Salas I, Villarreal-Martinez H. Spatial distribution of the larval indices of Aedes aegypti in Guadalupe, Nuevo Leon, Mexico, with circular distribution analysis. Journal of American Mosquito Control 19:15-18, 2003.

13. Morrison AC, Getis A, Santiago M. Exploratory space-time analysis of reported dengue cases during an outbreak in Florida, Puerto Rico, 1991 1992. American Journal of Tropical Medicine Hygiene 58: 287-298, 1992.

14. Pontes RJ, Freeman J, Oliveira-Lima JW, Hodgson JC, Spielman A. Vector densities that potentiate dengue outbreaks in a Brazilian city. The American Journal of Tropical Medicine and Hygiene 62: 378-383, 2000.

15. Souza-Santos R, Carvalho MS. Análise da distribuição espacial de Aedes aegypti na ilha do governador, Rio de Janeiro, Brasil. Cadernos de Saúde Pública 16: 109-118, 2000.

16. Taiul PL. Aspectos críticos do controle do dengue no Brasil. Cadernos de Saúde Pública 18: 867-871, 2002.

17. Teixeira GT, Barreto LB, Guerra Z. Epidemiologia e medidas de prevenção do dengue. Informe Epidemiológico do SUS 8: 5-33, 1999.

18. Vasconcelos PF, Lima JW, Raposo ML, Rodrigues SG, da Rosa JF, Amorim SM, da Rosa ES, Moura CM, Fonseca N, da Rosa AP. Inquérito sorológico na Ilha de São Luiz durante uma epidemia de dengue no Maranhão. Revista da Sociedade Brasileira de Medicina Tropical 32:171-179, 1999. 